\title{
Solutions of the Einstein Constraint Equations with Apparent Horizon Boundaries
}

\author{
David Maxwell \\ University of Washington
}

June 22, 2004

\begin{abstract}
We construct asymptotically Euclidean solutions of the vacuum Einstein constraint equations with an apparent horizon boundary condition. Specifically, we give sufficient conditions for the constant mean curvature conformal method to generate such solutions. The method of proof is based on the barrier method used by Isenberg for compact manifolds without boundary, suitably extended to accommodate semilinear boundary conditions and low regularity metrics. As a consequence of our results for manifolds with boundary, we also obtain improvements to the theory of the constraint equations on asymptotically Euclidean manifolds without boundary.
\end{abstract}

\section{Introduction}

The $N$-body problem in general relativity concerns the dynamics of an isolated system of $N$ black holes. One aspect of the problem, quite different from its classical counterpart, is the complexity of constructing appropriate initial data for the associated Cauchy problem. Initial data on an $n$-manifold $M$ is a Riemannian metric $g$ and a symmetric $(0,2)$-tensor $K$. We think of $M$ as an embedded spacelike hypersurface of an ambient Lorentz manifold $\mathcal{M} ; g$ is the pullback of the the Lorentz metric on $\mathcal{M}$ and $K$ is the extrinsic curvature of $M$ in $\mathcal{M}$. To model an isolated system of $N$ black holes in vacuum, the triple $(M, g, K)$ must satisfy several requirements. Isolated systems are typically modeled with asymptotically Euclidean initial data. This requires that $g$ approach the Euclidean metric and that $K$ decay to zero at far distances in $M$ (see Section 2 for a rigorous definition). Moreover, the vacuum Einstein equation imposes a compatibility condition on $K, g$, and its scalar curvature $R$

$$
\begin{array}{r}
R-|K|^{2}+\operatorname{tr} K^{2}=0 \\
\operatorname{div} K-d \operatorname{tr} K=0 .
\end{array}
$$

These are known as the Einstein constraint equations. Finally, data for $N$ black holes must evolve into a spacetime $\mathcal{M}$ containing an event horizon, and the intersection of the event horizon with $M$ must have $N$ connected components.

An event horizon is the boundary of the region that can send signals to infinity. It is a global property of a spacetime and cannot be located in an initial data set without evolving the data. This poses a serious obstacle to creating multiple black hole initial data. To address this problem, schemes such as those in [Mi63], [BL63], [YB80] and [Th87] (see also 
[Ck00]) create initial data containing an apparent horizon, defined below. The motivation for using apparent horizons comes from the weak Cosmic Censorship conjecture. Assuming that asymptotically Euclidean initial data evolves into a weakly censored spacetime, any apparent horizons present in the initial data will be contained in the black hole region of the spacetime. To generate spacetimes with multiple black holes, one constructs initial data with multiple apparent horizons. If the apparent horizons are well separated, one conjectures that they will be associated with distinct black holes.

An interesting approach to creating initial data containing apparent horizons, first suggested by Thornburg [Th87], is to work with a manifold with boundary and prescribe that the boundary be an apparent horizon. Thornburg numerically investigated generating such initial data. Variations of the apparent horizon condition have subsequently been proposed for numerical study, e.g. [Ck02] [Ea98]. However, as indicated by Dain [Da02], there has not been a rigorous mathematical investigation of the apparent horizon boundary condition. The goal of this paper is to take an initial step in addressing the problem of constructing asymptotically Euclidean Cauchy data satisfying the apparent horizon boundary condition. We exhibit sufficient conditions for generating a family of this data.

An apparent horizon is a surface that is instantaneously neither expanding nor contracting as it evolves under the flow of its outgoing (to infinity) orthogonal null geodesics. On the boundary of $M$, the expansion under this flow is given by the so-called convergence

$$
\theta_{+}=-\operatorname{tr} K+K(\nu, \nu)-(n-1) h,
$$

where $\nu$ is the exterior unit normal of $\partial M$ and $h$ is the mean curvature of $\partial M$ computed with respect to $-\nu$. Hence $\partial M$ is an apparent horizon if $\theta_{+}=0$. More generally, we say that $\partial M$ is outer marginally trapped if $\theta_{+} \leq 0$. The corresponding convergence for the incoming (from infinity) family of null geodesics is

$$
\theta_{-}=-\operatorname{tr} K+K(\nu, \nu)+(n-1) h .
$$

A surface is marginally trapped if both $\theta_{+} \leq 0$ and $\theta_{-} \leq 0$.

There is not a consistent definition of an apparent horizon in the literature. Other definitions of an apparent horizon include the boundary of a trapped region or an outermost marginally trapped surface. All these structures imply the existence of a black hole in the spacetime. We work with the definition $\theta_{+}=0$ since it is a local property and forms a natural boundary condition. Hence we seek asymptotically Euclidean data $(M, g, K)$ satisfying

$$
\begin{aligned}
R-|K|^{2}+\operatorname{tr} K^{2} & =0 \\
\operatorname{div} K-d \operatorname{tr} K & =0 \\
-\operatorname{tr} K+K(\nu, \nu)-(n-1) h & =0 \quad \text { on } \partial M .
\end{aligned}
$$


The conformal method of Lichnerowicz [Li44], Choquet-Bruhat and York [CBY80] provides a natural approach to the problem. For simplicity we work with its constant mean curvature (CMC) formulation, under which the constraint equations decouple. The conformal method seeks a solution of the form

$$
(\hat{g}, \hat{K})=\left(\phi^{\frac{4}{n-2}} g, \phi^{-2} \sigma+\frac{\tau}{n} g\right)
$$

where $g$ is a given asymptotically Euclidean metric prescribing the conformal class of $\hat{g}$, $\tau$ is a constant specifying tr $\hat{K}, \sigma$ is an unknown traceless symmetric $(0,2)$-tensor, and $\phi$ is an unknown conformal factor tending to 1 at infinity. From the decay conditions on $\hat{K}$ for asymptotically Euclidean initial data and the assumption that $\tau$ is constant, we have the further simplification $\tau=0$. Equations (4) then become a semilinear equation with semilinear boundary condition for $\phi$

$$
\begin{aligned}
-\Delta \phi+\frac{1}{a}\left(R \phi-|\sigma|^{2} \phi^{-3-2 \kappa}\right) & =0 \\
\partial_{\nu} \phi+\frac{1}{\kappa} h \phi-\frac{2}{a} \sigma(\nu, \nu) \phi^{-1-\kappa} & =0 \quad \text { on } \partial M
\end{aligned}
$$

and a linear system for $\sigma$

$$
\operatorname{div} \sigma=0 \text {. }
$$

The first equation of (5) is known as the Lichnerowicz equation. In (5), the dimensional constants are $\kappa=2 /(n-2)$ and $a=2 \kappa+4$. Note that we use the exterior normal $\nu$ to follow traditional PDE notation, but the mean curvature $h$ is computed with respect to the interior unit normal.

A trace-free, symmetric (0,2)-tensor $\sigma$ satisfying (6) is called transverse traceless. The set of transverse traceless tensors forms a linear space, and the choice of $\sigma$ can be thought of as data to be prescribed in solving (5). Hence, we wish to find conditions on $g$ and $\sigma$ under which (5) is solvable.

The construction of solutions of (5) starts with an asymptotically Euclidean manifold $\left(M, g^{\prime}\right)$ satisfying

$$
\lambda_{g^{\prime}}>0
$$

For any asymptotically Euclidean metric $(M, g), \lambda_{g}$ is the conformal invariant

$$
\lambda_{g}=\inf _{f \in C_{\mathrm{c}}^{\infty}(M), f \neq 0} \frac{\int_{M} a|\nabla f|^{2}+R f^{2} d V+\int_{\partial M} \frac{a}{\kappa} h f^{2} d A}{\|f\|_{L^{2 n /(n-2)}}^{2}} .
$$

This is analogous to an invariant for compact manifolds with boundary found in [Es92]. We next make a conformal change from $g^{\prime}$ to a metric $g$ satisfying $R=0$ and $h<0$; 
Corollary 4.2 ensures this can always be done. Our main result, Theorem 4.3 proves that (5) is then solvable if

$$
(n-1) h \leq \sigma(\nu, \nu) \leq 0
$$

We prove Theorem 4.3 using a barrier method for semilinear boundary conditions. Section 3 establishes a general existence theorem and Section 4 applies it to system (5).

One can easily find asymptotically Euclidean manifolds with boundary that satisfy $\lambda_{g}>0$. For example, every manifold with $R \geq 0$ and $h \geq 0$ has $\lambda_{g}>0$. So condition (7) of the construction can be readily met. On the other hand, it is not obvious that the restriction (8) is reasonable. In Section 5 we show that on any sufficiently smooth asymptotically Euclidean manifold with boundary we can freely specify $\sigma(\nu, \nu)$ on the boundary. That is, given a function $f$ on $\partial M$, we can find a transverse traceless tensor $\sigma$ satisfying $\sigma(\nu, \nu)=f$. This follows from the solution to a boundary value problem for the vector Laplacian. Since $h<0$, it follows that there exists a large family of transverse traceless tensors $\sigma$ satisfying (8).

It should be noted that our construction is not a full parameterization of the set of CMC solutions of (4). Since condition (8) is not conformally invariant, the set of allowed transverse traceless tensors depends on the choice of conformal representative satisfying $R=0$ and $h<0$. Hence condition (8) is not necessary. Moreover, although technical condition (7) is vital for the construction, it is not clear if it is necessary. Hence there remains much to be understood about parameterizing the full set of solutions.

In light of recent low regularity a priori estimates for solutions of the evolution problem [KR02] [ST], there is interest in generating low regularity solutions of the constraints. In terms of $L^{p}$ Sobolev spaces, a natural setting is $(g, \sigma) \in W_{\mathrm{loc}}^{2, \frac{n}{2}+\epsilon} \times W_{\mathrm{loc}}^{1, \frac{n}{2}+\epsilon}$. This is the weakest regularity that ensures that $g$ has curvature in an $L^{p}$ space and that the Sobolev space containing $g$ is an algebra. Y. Choquet-Bruhat has announced [CB03] a construction of such low regularity solutions of the constraint equations in the context of compact manifolds. We construct asymptotically Euclidean solutions with this level of regularity, but under a possibly unneeded assumption. In order to find suitable transverse traceless tensors, we require that $(M, g)$ not admit any nontrivial conformal Killing fields vanishing at infinity. This is known to be true [CO81] for $C^{3}$ asymptotically Euclidean manifolds. We prove this also holds for metrics with regularity as weak as $W^{2, n+\epsilon}$. To consider $W^{2, \frac{n}{2}+\epsilon}$ metrics, however, we must assume the non-existence of such fields.

In a previous version of this article, we constructed solutions of (4) under the hypotheses $\lambda_{g}>0$ and $\sigma(\nu, \nu) \geq 0$. These solutions have the undesirable feature that although the boundary is a outer marginally trapped surface, it is not a marginally trapped surface. This observation was made in [Da03], which appeared shortly after our results were announced. 
From (2) and (3) we see that $\hat{\theta}_{-}=\hat{\theta}_{+}+2(n-1) \hat{h}$. Since $\hat{\theta}_{+}=0$ on $\partial M$, we have $\hat{\theta}_{-}=2(n-1) \hat{h}$. So $\partial M$ is a marginally trapped surface if and only if $\hat{h} \leq 0$. The sign of $\hat{h}$ is determined by $\sigma(\nu, \nu)$ since $\hat{\theta}_{+}=0$ implies

$$
(n-1) \hat{h}=\hat{K}(\hat{\nu}, \hat{\nu})=\phi^{-2-2 \kappa} \sigma(\nu, \nu) .
$$

So $\hat{h} \leq 0$ if and only if $\sigma(\nu, \nu) \leq 0$. Under the hypothesis $\sigma(\nu, \nu) \geq 0$, one can construct an apparent horizon that is also a marginally trapped surface only if $\sigma(\nu, \nu)=0$, which leads to $\hat{\theta}_{-}=0$ and $\hat{h}=0$.

In [Da03], Dain constructs trapped surface boundaries by working with $\hat{\theta}_{-}$rather than $\hat{\theta}_{+}$. Under suitable hypotheses, [Da03] prescribes $\hat{\theta}_{-} \leq 0$ and constructs boundaries with $\hat{\theta}_{+} \leq \hat{\theta}_{-} \leq 0$. These are trapped surfaces, but the inequality $\hat{\theta}_{+} \leq \hat{\theta}_{-}$shows that the resulting boundaries satisfy $\hat{h} \geq 0$. In particular, the techniques of [Da03] also cannot construct a boundary that is simultaneously a marginally trapped surface and an apparent horizon unless $\hat{\theta}_{-}=0$ and $\hat{h}=0$.

In the current version of this article, we construct solutions with $\hat{h} \leq 0$. To do this requires we assume $\sigma(\nu, \nu) \leq 0$, since the sign of $\hat{h}$ is determined by the sign of $\sigma(\nu, \nu)$. The resulting PDEs are more delicate, and the hypothesis $(n-1) h \leq \sigma(\nu, \nu)$ arises to compensate for this. Since $\hat{h} \leq 0$, the apparent horizons we construct satisfy $\hat{\theta}_{-} \leq \hat{\theta}_{+}=0$, with strict inequality wherever $\sigma(\nu, \nu)$ (and hence $\hat{h}$ ) is negative. Figure 1 shows boundary mean curvatures of various signs and further indicates the naturality of the condition $\hat{h} \leq 0$.

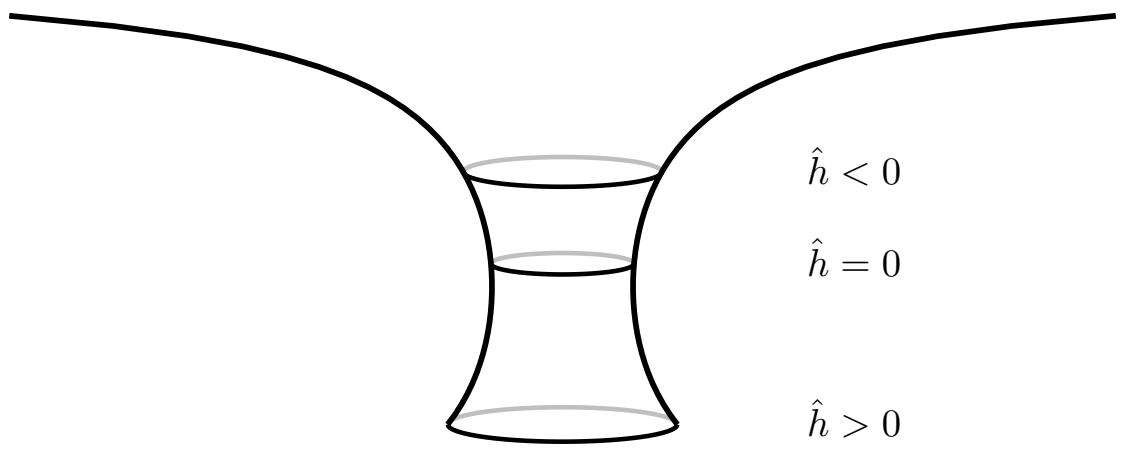

Figure 1: Boundary mean curvatures of an asymptotically Euclidean manifold.

\subsection{Notation}

Let $\mathcal{M}$ be a Lorentz manifold with metric $\gamma$ and connection $D$. The signature of $\gamma$ is $(-+\cdots+)$. If $M$ is a spacelike hypersurface of $\mathcal{M}$ with timelike unit normal $N$, we define the extrinsic curvature $K$ of $M$ in $\mathcal{M}$ by $K(X, Y)=\left\langle D_{X} Y, N\right\rangle_{\gamma}$ for vector fields tangent 
to $M$. This definition agrees with that used in [YB80] and [Da02], but differs in sign from that used in [Wa84] and [Da03].

Let $M$ be a Riemannian manifold with metric $g$ and connection $\nabla$, If $\Sigma$ is a spacelike hypersurface if $M$ with unit normal $\nu$, the extrinsic curvature $k$ of $\Sigma$ in $M$ is similarly defined by $k(X, Y)=\left\langle\nabla_{X} Y, \nu\right\rangle_{g}$. The mean curvature $h$ of $\Sigma$ computed with respect to $\nu$ is $\frac{1}{n-1} \operatorname{tr} k$, where $n$ is the dimension of $M$.

Throughout this paper we take $n$ to be a fixed integer with $n \geq 3$ and $\rho$ to be a negative real number. If $p \in[1, \infty]$, we define the critical Sobolev exponent $p^{*}=\frac{n p}{n-p}$ if $p<n$ and we set $p^{*}=\infty$ otherwise. The ball of radius $r$ about $x$ is $B_{r}(x)$, and $E_{r}$ is the region exterior to $\bar{B}_{r}(0)$. We define $f^{(+)}(x)=\max (f(x), 0)$.

We use the notation $A \lesssim B$ to mean $A<c B$ for a certain positive constant $c$. The constant is independent of the functions and parameters appearing in $A$ and $B$ that are not assumed to have a fixed value. For example, when considering a sequence $\left\{f_{i}\right\}_{i=1}^{\infty}$ of functions on a domain $\Omega$, the expression $\left\|f_{i}\right\|_{L^{1}(\Omega)} \lesssim 1$ means the sequence is uniformly bounded in $L^{1}(\Omega)$ (with a bound that might depend on $\Omega$ ).

\section{Asymptotically Euclidean Manifolds}

An asymptotically Euclidean manifold is a non-compact Riemannian manifold, possibly with boundary, that can be decomposed into a compact core and a finite number of ends where the metric approaches the Euclidean metric at far distances. To make this loose description precise, we use weighted function spaces that prescribe asymptotic behavior like $|x|^{\delta}$ for large $x$. For $x \in \mathbb{R}^{n}$, let $w(x)=\left(1+|x|^{2}\right)^{1 / 2}$. Then for any $\delta \in \mathbb{R}$ and and any open set $\Omega \subset \mathbb{R}^{n}$, the weighted Sobolev space $W_{\delta}^{k, p}(\Omega)$ is the subset of $W_{\text {loc }}^{k, p}(\Omega)$ for which the norm

$$
\|u\|_{W_{\delta}^{k, p}(\Omega)}=\sum_{|\beta| \leq k}\left\|w^{-\delta-\frac{n}{p}+|\beta|} \partial^{\beta} u\right\|_{L^{p}(\Omega)}
$$

is finite; we will always work with spaces for which $p \neq 1, \infty$. Weighted spaces of continuous functions are defined by the norm

$$
\|u\|_{C_{\delta}^{k}(\Omega)}=\sum_{|\alpha| \leq k} \sup _{x \in \Omega} w(x)^{-\delta+|\alpha|}\left|\partial^{\alpha} u(x)\right| .
$$

Our indexing convention for $\delta$ follows [Ba86] so that the value of $\delta$ directly encodes asymptotic growth at infinity. We refer the reader to [Ba86] for properties of these weighted spaces. In particular, we recall the following facts.

\section{Lemma 2.1.}

1. If $p \leq q$ and $\delta^{\prime}<\delta$ then $L_{\delta^{\prime}}^{p}(\Omega) \subset L_{\delta}^{q}(\Omega)$ and the inclusion is continuous. 
2. For $k \geq 1$ and $\delta^{\prime}<\delta$ the inclusion $W_{\delta^{\prime}}^{k, p}(\Omega) \subset W_{\delta}^{k-1, p}(\Omega)$ is compact.

3. If $1 / p>k / n$ then $W_{\delta}^{k, p}(\Omega) \subset L_{\delta}^{r}(\Omega)$ where $1 / r=1 / p-k / n$. If $1 / p=k / n$ then $W_{\delta}^{k, p}(\Omega) \subset L_{\delta}^{r}(\Omega)$ for all $r \geq p$. If $1 / p<k / n$ then $W_{\delta}^{k, p}(\Omega) \subset C_{\delta}^{0}(\Omega)$. These inclusions are continuous.

4. If $m \leq \min (j, k), p \leq q, \epsilon>0$, and $1 / q<(j+k-m) / n$, then multiplication is a continuous bilinear map from $W_{\delta_{1}}^{j, q}(\Omega) \times W_{\delta_{2}}^{k, p}(\Omega)$ to $W_{\delta_{1}+\delta_{2}+\epsilon}^{m, p}(\Omega)$. In particular, if $1 / p<k / n$ and $\delta<0$, then $W_{\delta}^{k, p}(\Omega)$ is an algebra.

Let $M$ be a smooth, connected, $n$-dimensional manifold with boundary, and let $g$ be a metric on $M$ for which $(M, g)$ is complete, and let $\rho<0$ (these will be standing assumptions for the remainder of the paper). We say $(M, g)$ is asymptotically Euclidean of class $W_{\rho}^{k, p}$ if:

i. The metric $g \in W_{\text {loc }}^{k, p}(M)$, where $1 / p-k / n<0$ (and consequently $g$ is continuous).

ii. There exists a finite collection $\left\{N_{i}\right\}_{i=1}^{m}$ of open subsets of $M$ and diffeomorphisms $\Phi_{i}: E_{1} \mapsto N_{i}$ such that $M-\cup_{i} N_{i}$ is compact.

iii. For each $i, \Phi_{i}^{*} g-\bar{g} \in W_{\rho}^{k, p}\left(E_{1}\right)$.

We call the charts $\Phi_{i}$ end charts and the corresponding coordinates are end coordinates. Suppose $(M, g)$ is asymptotically Euclidean, and let $\left\{\Phi_{i}\right\}_{i=1}^{m}$ be its collection of end charts. Let $K=M-\cup_{i} \Phi_{i}\left(E_{2}\right)$, so $K$ is a compact manifold with boundary. The weighted Sobolev space $W_{\delta}^{k, p}(M)$ is the subset of $W_{\text {loc }}^{k, p}(M)$ such that the norm

$$
\|u\|_{W_{\delta}^{k, p}(M)}=\|u\|_{W^{k, p}(K)}+\sum_{i}\left\|\Phi_{i}^{*} u\right\|_{W_{\delta}^{k, p}\left(E_{1}\right)}
$$

is finite. The weighted spaces $L_{\delta}^{p}(M)$ and $C_{\delta}^{k}(M)$ are defined similarly, and we let $C_{\delta}^{\infty}(M)=\cap_{k=0}^{\infty} C_{\delta}^{k}(M)$. Lemma 2.1 applies equally well to asymptotically Euclidean manifolds.

Using these weighted spaces we can now define an asymptotically Euclidean data set. The extrinsic curvature tensor $K$ of an initial data set $(M, g, K)$ should behave like a first derivative of $g$. Hence, if $(M, g)$ is asymptotically Euclidean of class $W_{\rho}^{k, p}$, we say $(M, g, K)$ is an asymptotically Euclidean data set if $K \in W_{\rho-1}^{k-1, p}(M)$.

\section{Barrier Method For Semilinear Boundary Conditions}

In [Is95], Isenberg used a constructive barrier method (also known as the method of suband supersolutions) to completely parameterize $\mathrm{CMC}$ solutions of the constraint equations on a compact manifold. Subsequently, the method has been applied to construct non-CMC 
solutions on compact manifolds [MI96] and asymptotically Euclidean solutions [CBIY00]. In this section we provide a version of the generic barrier construction that accommodates semilinear boundary conditions and low regularity metrics.

Consider the boundary value problem

$$
\begin{aligned}
-\Delta u & =F(x, u) \\
\partial_{\nu} u & =f(x, u) \quad \text { on } \partial M
\end{aligned}
$$

on an asymptotically Euclidean manifold. We use the convention that $\Delta$ has negative eigenvalues, so $\Delta=\partial_{x_{1}}^{2}+\cdots+\partial_{x_{n}}^{2}$ in Euclidean space. A subsolution of equation (9) is a function $u_{-}$that satisfies

$$
\begin{aligned}
-\Delta u_{-} & \leq F\left(x, u_{-}\right) \\
\partial_{\nu} u_{-} & \leq f\left(x, u_{-}\right) \quad \text { on } \partial M
\end{aligned}
$$

and a supersolution $u_{+}$is defined similarly with the inequalities reversed. In Proposition 3.5 below, we show that if there exists a subsolution $u_{-}$and a supersolution $u_{+}$decaying at infinity and satisfying $u_{-} \leq u_{+}$, then there exists a solution $u$ satisfying $u_{-} \leq u \leq u_{+}$.

The proof of Proposition 3.5 relies on properties of the associated linearized operator

$$
\begin{aligned}
-\Delta u+V u & =F \\
\partial_{\nu} u+\mu u & =f, \quad \text { on } \partial M
\end{aligned}
$$

where $V, \mu, F$, and $f$ are functions of $x$ alone. Let $\mathcal{P}$ denote $\left((-\Delta+V),\left.\left(\partial_{\nu}+\mu\right)\right|_{\partial M}\right)$.

Proposition 3.1. Suppose $(M, g)$ is asymptotically Euclidean of class $W_{\rho}^{k, p}, k \geq 2, k>$ $n / p$, and suppose $V \in W_{\rho-2}^{k-2, p}$ and $\mu \in W^{k-1-\frac{1}{p}, p}$. Then if $2-n<\delta<0$ the operator $\mathcal{P}: W_{\delta}^{k, p}(M) \rightarrow W_{\delta-2}^{k-2, p}(M) \times W^{k-1-\frac{1}{p}, p}(\partial M)$ is Fredholm with index 0. Moreover, if $V \geq 0$ and $\mu \geq 0$ then $\mathcal{P}$ is an isomorphism.

In Proposition 5.3 we prove a similar result for the vector Laplacian. Since the details are tedious and largely similar, we omit the proof of Proposition 3.1. The only substantial difference from the proof of Proposition 5.3 is the method used to show $\mathcal{P}$ is injective when $V$ and $\mu$ are nonnegative. This is an easy consequence of the following weak maximum principle.

Lemma 3.2. Suppose $(M, g), V$, and $\mu$ satisfy the hypotheses of Proposition 3.1 and suppose $V \geq 0$ and $\mu \geq 0$. If $u \in W_{\mathrm{loc}}^{k, p}$ satisfies

$$
\begin{aligned}
-\Delta u+V u & \leq 0 \\
\partial_{\nu} u+\mu u & \leq 0, \quad \text { on } \partial M
\end{aligned}
$$


and if $u^{(+)}$is $o(1)$ on each end of $M$, then $u \leq 0$. In particular, if $u \in W_{\delta}^{k, p}(M)$ for some $\delta<0$ and $u$ satisfies (11), then $u \leq 0$.

Proof: Fix $\epsilon>0$, and let $v=(u-\epsilon)^{(+)}$. Since $u^{(+)}=o(1)$ on each end, we see $v$ is compactly supported. Moreover, since $u \in W_{\text {loc }}^{k, p}$ we have from Sobolev embedding that $u \in W_{\mathrm{loc}}^{1,2}$ and hence $v \in W^{1,2}$. Now,

$$
\int_{M}-v \Delta u d V \leq-\int_{M} V u v d V \leq 0
$$

since $V \geq 0, v \geq 0$ and since $u$ is positive wherever $v \neq 0$. Integrating by parts we have

$$
\int_{M}|\nabla v|^{2} d V-\int_{\partial M} v \partial_{\nu} u d V \leq 0
$$

since $\nabla u=\nabla v$ on the support of $v$. From the boundary condition we obtain

$$
\int_{M}|\nabla v|^{2} d V \leq-\int_{\partial M} \mu u v d V \leq 0
$$

since $\mu \geq 0$. So $v$ is constant and compactly supported, and we conclude $u \leq \epsilon$. Sending $\epsilon$ to 0 proves $u \leq 0$.

Finally, if $u \in W_{\delta}^{k, p}$, then $u \in C_{\delta}^{0}$. Hence if $\delta<0$, then $u^{(+)}=o(1)$ and the lemma can be applied to $u$.

If $V$ or $\mu$ is negative at some point, the kernel of $\mathcal{P}$ might not be empty. We have the following estimate for how elements of the kernel decay at infinity.

Lemma 3.3. Suppose $(M, g), V$, and $\mu$ satisfy the hypotheses of Proposition 3.1 and suppose that $u \in W_{\delta}^{k, p}$ with $\delta<0$ is in the kernel of $\mathcal{P}$. Then $u \in W_{\delta^{\prime}}^{k, p}(M)$ for every $\delta^{\prime} \in(2-n, 0)$.

Proof: Since $V \in W_{\rho-2}^{k-2, p}$ we have $V u \in W_{\rho+\delta-2}^{k-2, p}$. Hence

$$
\begin{aligned}
\left(-\Delta u, \partial_{\nu} u\right) & =(-V u,-\mu u) \\
& \in W_{\rho+\delta-2}^{k-2, p}(M) \times W^{k-1-\frac{1}{p}, p}(\partial M) .
\end{aligned}
$$

Since $\left(-\Delta,\left(\left.\partial_{\nu}\right|_{\partial M}\right)\right)$ is an isomorphism on $W_{\delta^{\prime}}^{k, p}$ for each $\delta^{\prime} \in(2-n, 0)$, we conclude that $u \in W_{\delta^{\prime}}^{k, p}$ for each $\delta^{\prime} \in(\max (2-n, \rho+\delta-2), 0)$. Iterating this argument a finite number of times yields the desired result.

Although the barrier construction in Proposition 3.5 below only uses the weak maximum principle Lemma 3.2, we need the following strong maximum principle in our later analysis of the Lichnerowicz equation to ensure that the conformal factors we construct never vanish. Note that there is no sign restriction on $V$ and $\mu$. 
Lemma 3.4. Suppose $(M, g), V$, and $\mu$ satisfy the hypotheses of Proposition 3.1. Suppose also that $u \in W_{\mathrm{loc}}^{k, p}(M)$ is nonnegative and satisfies

$$
\begin{aligned}
-\Delta u+V u & \geq 0 \\
\partial_{\nu} u+\mu u & \geq 0 \quad \text { on } \partial M .
\end{aligned}
$$

If $u(x)=0$ at some point $x \in M$, then $u$ vanishes identically.

Proof: From Sobolev embedding, we can assume without loss of generality that the hypotheses of Proposition 3.1 are satisfied with $k=2$. Suppose first that $x$ is an interior point of $M$. Since $g$ is continuous and $V \in L_{\mathrm{loc}}^{p}(M)$ with $p>n / 2$, the weak Harnack inequality of [Tr73] holds and we have for some radius $R$ sufficiently small and some exponent $q$ sufficiently large there exists a constant $C>0$ such that

$$
\|u\|_{L^{q}\left(B_{2 R}(x)\right.} \leq C \inf _{B_{R}(x)} u=0
$$

Hence $u$ vanishes in a neighbourhood of $x$, and a connectivity argument implies $u$ is identically 0 .

It remains to consider the case where $u$ vanishes at a point $x \in \partial M$. Working in local coordinates about $x$ we can do our analysis on $B_{1}^{+}(0) \equiv B_{1}(0) \cap \mathbb{R}_{+}^{n}$, where balls are now taken with respect to the flat background metric. Let $b$ be a $W^{1, p}\left(B_{1}^{+}\right)$vector field such that $\langle b, \nu\rangle=\mu$ on $D_{1}$, where $D_{1}$ is the flat portion of the boundary of $B_{1}^{+}$. For example, since $\mu \in W^{1-\frac{1}{p}, p}$ and $g \in W_{\text {loc }}^{2, p}$, we can take $b=\hat{\mu} \hat{\nu}$ where $\hat{\mu}$ is a $W^{1, p}$ extension of $\mu$ and $\hat{\nu}$ is a $W^{2, p}$ extension of $\nu$. Integrating by parts, we have for any nonnegative $\phi \in C_{\mathrm{c}}^{\infty}\left(B_{1}^{+} \cup D_{1}\right)$

$$
\int_{B_{1}^{+}}\langle\nabla \phi, b\rangle u+u \phi \operatorname{div} b+\langle b, \nabla u\rangle \phi d V=\int_{D_{1}} \mu u \phi d A
$$

Hence

$$
\begin{aligned}
& \int_{B_{1}^{+}}\langle\nabla u, \nabla \phi\rangle_{g}+R u \phi+ \\
&+\langle\nabla \phi, b\rangle u+u \phi \operatorname{div} b+\langle b, \nabla u\rangle \phi d V=\int_{B_{1}^{+}}-\phi \Delta u+R u \phi d V+ \\
&+\int_{D_{1}} \partial_{\nu} u \phi+\mu \phi u d A \\
& \geq 0,
\end{aligned}
$$

since $u$ is a supersolution.

To reduce to the interior case, we now construct an elliptic equation on all of $B_{1}$. For any function or tensor $f$ defined on $B_{1}^{+}(0)$, let $\tilde{f}$ be the extension of $f$ to $B_{1}$ via its pushforward 
under reflection. It follows from (12) and a change of variables argument that for any nonnegative $\phi \in C_{\mathrm{c}}^{\infty}\left(B_{1}\right)$,

$$
\int_{B_{1}}\langle\nabla \tilde{u}, \nabla \phi\rangle_{\tilde{g}}+\tilde{R} \tilde{u} \phi+\langle\nabla \phi, \tilde{b}\rangle_{\tilde{g}} \tilde{u}+\tilde{u} \phi \overparen{\operatorname{div} b}+\langle\tilde{b}, \nabla \tilde{u}\rangle_{\tilde{g}} \phi \widetilde{d V} \geq 0 .
$$

Since $\tilde{g} \in W^{1,2 p}\left(B_{1}\right), \tilde{R} \in L^{p}\left(B_{1}\right), \tilde{b} \in L^{2 p}\left(B_{1}\right)$, and $\overparen{\operatorname{div} b} \in L^{p}\left(B_{1}\right)$, we conclude from (13) that $\tilde{u}$ is a weak $W^{1,2 p}$ supersolution of an elliptic equation with coefficients having regularity considered by [Tr73]. Since $\tilde{u} \geq 0$ and $\tilde{u}(0)=0$, the weak Harnack inequality again implies that $u$ vanishes in a neighbourhood of $x$ and hence on all of $M$.

We now turn to the existence proof for the nonlinear problem (9). We assume for simplicity that the nonlinearities $F$ and $f$ have the form

$$
\begin{aligned}
F(x, y) & =\sum_{j=1}^{l} F_{j}(x) G_{j}(y) \\
f(x, y) & =\sum_{j=1}^{m} f_{j}(x) g_{j}(y) .
\end{aligned}
$$

\section{Proposition 3.5. Suppose}

1. $(M, g)$ is asymptotically Euclidean of class $W_{\rho}^{k, p}$ with $k \geq 2, p>n / k$, and $\rho<0$,

2. $u_{-}, u_{+} \in W_{\delta}^{k, p}$ with and $\delta \in(2-n, 0)$ are a subsolution and a supersolution respectively of (9) such that $u_{-} \leq u_{+}$,

3. each $F_{j} \in W_{\delta-2}^{k-2, p}(M)$ and $f_{j} \in W^{k-1-\frac{1}{p}, p}(\partial M)$,

4. each $G_{j}$ and $g_{j}$ are smooth on $I=\left[\inf \left(u_{-}\right), \sup \left(u_{+}\right)\right]$.

Then there exists a solution $u$ of (9) such that $u_{-} \leq u \leq u_{+}$.

Proof: $\quad$ We first assume $k=2$ and $p>n / 2$. Let

$$
\begin{gathered}
V(x)=\sum_{j=1}^{l}\left|F_{j}(x)\right|\left|\min _{I} G_{j}^{\prime}\right| \\
\mu(x)=\sum_{j=1}^{m}\left|f_{j}(x)\right|\left|\min _{I} g_{j}^{\prime}\right|,
\end{gathered}
$$

so that $V \in L_{\delta-2}^{p}(M), \mu \in W^{1-\frac{1}{p}, p}(\partial M)$, and both are nonnegative. Let $F_{V}(x, y)=$ $F(x, y)+V(x) y$ and $f_{\mu}(x, y)=f(x, y)+\mu(x) y$ so that $F_{V}$ and $f_{\mu}$ are both non-decreasing in $y$. Let $L_{V}=-\Delta+V$, and let $B_{\mu}=\left.\left(\partial_{\nu}+\mu\right)\right|_{\partial M}$. From Proposition 3.1 we have $\left(L_{V}, B_{\mu}\right)$ is an isomorphism acting on $W_{\delta}^{2, p}$. 
We construct a monotone decreasing sequence of functions $u_{+}=u_{0} \geq u_{1} \geq u_{2} \geq \cdots$ by letting

$$
\begin{aligned}
L_{V} u_{i+1} & =F_{V}\left(x, u_{i}\right) \\
B_{\mu} u_{i+1} & =f_{\mu}\left(x, u_{i}\right) .
\end{aligned}
$$

The monotonicity of the sequence follows from the maximum principle and the monotonicity of $F_{V}(x, y)$ and $f_{\mu}(x, y)$ in $y$. The maximum principle also implies $u_{i} \geq u_{-}$.

We claim the sequence $\left\{u_{i}\right\}_{i=1}^{\infty}$ is bounded in $W_{\delta}^{2, p}(M)$. From Proposition 3.1 we can estimate

$$
\left\|u_{i+1}\right\|_{W_{\delta}^{2, p}(M)} \lesssim\left\|F_{V}\left(x, u_{i}\right)\right\|_{L_{\delta-2}^{p}(M)}+\left\|f_{\mu}\left(x, u_{i}\right)\right\|_{W^{1-\frac{1}{p}, p}(\partial M)} \cdot
$$

Pick $q \in(p, \infty)$ such that

$$
\frac{1}{p}-\frac{1}{n}<\frac{1}{q}<\frac{1}{n}
$$

which is possible since $p>n / 2$. Then

$$
\left\|F_{V}\left(x, u_{i}\right)\right\|_{L_{\delta-2}^{p}(M)}+\left\|f_{\mu}\left(x, u_{i}\right)\right\|_{W^{1-\frac{1}{p}, p}(\partial M)} \lesssim 1+\left\|u_{i}\right\|_{W^{1, q}(U)}
$$

for any fixed smooth bounded neighbourhood $U$ of $\partial M$. From interpolation and Sobolev embedding we have for any $\epsilon>0$

$$
\left\|u_{i}\right\|_{W^{1, q}(U)} \lesssim C(\epsilon)\left\|u_{i}\right\|_{W^{1, p}(U)}+\epsilon\left\|u_{i}\right\|_{W^{2, p}(U)} .
$$

A second application of interpolation then implies

$$
\left\|u_{i}\right\|_{W^{1, q}(U)} \lesssim C(\epsilon)\left\|u_{i}\right\|_{L^{p}(U)}+\epsilon\left\|u_{i}\right\|_{W^{2, p}(U)} \cdot
$$

Hence

$$
\left\|u_{i}\right\|_{W^{1, q}(U)} \lesssim C(\epsilon)\left\|u_{i}\right\|_{L_{\delta}^{p}(M)}+\epsilon\left\|u_{i}\right\|_{W_{\delta}^{2, p}(M)} \cdot
$$

Since $u_{-} \leq u_{i} \leq u_{+}$, we have $\left\|u_{i}\right\|_{L_{\delta}^{p}(M)}$ is uniformly bounded. Combining (14), (15) and (16) we obtain, taking $\epsilon$ sufficiently small,

$$
\left\|u_{i+1}\right\|_{W_{\delta}^{2, p}} \leq \frac{1}{2}\left\|u_{i}\right\|_{W_{\delta}^{2, p}}+C
$$

Iterating this inequality we obtain a bound for all $i$

$$
\left\|u_{i}\right\|_{W_{\delta}^{2, p}} \leq\left\|u_{+}\right\|_{W_{\delta}^{2, p}}+2 C
$$

Hence some subsequence of $\left\{u_{i}\right\}_{i=1}^{\infty}$ (and by monotonicity, the whole sequence) converges weakly in $W_{\delta}^{2, p}$ to a limit $u_{\infty}$. 
It remains to see $u_{\infty}$ is a solution of (9). Now $u_{i}$ converges strongly to $u_{\infty}$ in $W_{\delta^{\prime}}^{1, p}$ for any $\delta^{\prime}>\delta$, and also converges uniformly on compact sets. Hence for any $\phi \in C_{\mathrm{c}}^{\infty}(M)$,

$$
\begin{aligned}
\int_{M}\left(F_{V}\left(x, u_{i}\right)-V(x) u_{i+1}\right) \phi d V & \rightarrow \int_{M} F\left(x, u_{\infty}\right) \phi d V \\
\int_{\partial M}\left(f_{\mu}\left(x, u_{i}\right)-\mu(x) u_{i+1}\right) \phi d A & \rightarrow \int_{\partial M} f\left(x, u_{\infty}\right) \phi d A \\
\int_{M}\left\langle\nabla u_{i+1}, \nabla \phi\right\rangle \phi d V & \rightarrow \int_{M}\left\langle\nabla u_{\infty}, \nabla \phi\right\rangle d V .
\end{aligned}
$$

So

$$
\int_{M}\left\langle\nabla u_{\infty}, \nabla \phi\right\rangle d V=\int_{M} F\left(x, u_{\infty}\right) \phi d V+\int_{\partial M} f\left(x, u_{\infty}\right) \phi d A,
$$

and an application of integration by parts shows $u_{\infty}$ solves the boundary value problem.

The case $k>2$ now follows from a bootstrap using the previous result together with Proposition 3.1 .

\section{Solving the Lichnerowicz Equation}

We now prove the existence of solutions of the Lichnerowicz equation

$$
\begin{aligned}
-\Delta \phi+\frac{1}{a}\left(R \phi-|\sigma|^{2} \phi^{-3-2 \kappa}\right) & =0 \\
\partial_{\nu} \phi+\frac{1}{\kappa} h \phi-\frac{2}{a} \sigma(\nu, \nu) \phi^{-1-\kappa} & =0 \quad \text { on } \partial M .
\end{aligned}
$$

We first show that if $\lambda_{g^{\prime}}>0$, then $\left(M, g^{\prime}\right)$ is conformally equivalent to $(M, g)$, where $g$ satisfies $\lambda_{g}>0, R=0$ and $h<0$. We then show that if $\lambda_{g}>0, R=0$, and $(n-1) h \leq \sigma(\nu, \nu) \leq 0$, then there exist a sub/supersolution pair for (17) and we apply Proposition 3.5 to obtain a solution.

The following proposition gives useful conditions equivalent to $\lambda_{g}>0$. We define for compactly supported functions

$$
Q_{g}(f)=\frac{\int_{M} a|\nabla f|^{2}+R f^{2} d V+\int_{\partial M} \frac{a}{\kappa} h f^{2} d A}{\|f\|_{L^{2^{*}}}^{2}},
$$

Thus

$$
\lambda_{g}=\inf _{f \in C_{\mathrm{c}}^{\infty}(M), f \neq 0} Q_{g}(f)
$$

We also define

$$
\mathcal{P}_{\eta}=\left(\left(-\Delta+\frac{\eta}{a} R\right),\left.\left(\partial_{\nu}+\frac{\eta}{\kappa} h\right)\right|_{\partial M}\right)
$$

If $k \geq 2, \delta<0$, and $k>n / p$, then $\mathcal{P}_{\eta}$ is a continuous map from $W_{\delta}^{k, p}(M)$ to $W_{\delta-2}^{k-2, p}(M) \times$ $W^{k-1-\frac{1}{p}, p}(\partial M)$. 
Proposition 4.1. Suppose $(M, g)$ is asymptotically Euclidean of class $W_{\delta}^{k, p}, k \geq 2, k>$ $n / p$, and $2-n<\delta<0$. Then the following conditions are equivalent:

1. There exists a conformal factor $\phi>0$ such that $1-\phi \in W_{\delta}^{k, p}$ and such that $\left(M, \phi^{2 \kappa} g\right)$ is scalar flat and has a minimal surface boundary.

2. $\lambda_{g}>0$.

3. $\mathcal{P}_{\eta}$ is an isomorphism acting on $W_{\delta}^{k, p}$ for each $\eta \in[0,1]$.

Proof: Suppose condition 1 holds. Since $\lambda_{g}$ is a conformal invariant, we can assume that $R=0$ and $h=0$. We first make a conformal change to a metric with positive scalar curvature. Let $\mathcal{R}$ be any continuous positive element of $W_{\delta-2}^{k-2, p}$ and let $v$ be the unique solution given by Proposition 3.1 to

$$
\begin{array}{r}
-\Delta_{\tilde{g}} v=\frac{1}{a} \mathcal{R} \\
\partial_{\nu} v=0 .
\end{array}
$$

The maximum principle implies $v \geq 0$, and hence $\phi=1+v>0$. Letting $\hat{g}=\phi^{2 \kappa} g$, it follows that $\hat{R}$ is positive and continuous, and that $\hat{h}=0$. From Sobolev embedding and a standard argument with cutoff functions, we have

$$
\|f\|_{L^{2^{*}}}^{2} \lesssim\|\nabla f\|_{L^{2}}^{2}+\|f\|_{L^{2}(K)}^{2}
$$

where $K$ is the compact core of $M$. Since $\hat{R}$ is bounded below on $K$ we find

$$
\|f\|_{L^{2^{*}}}^{2} \lesssim\|\nabla f\|_{L^{2}}^{2}+\left\|\hat{R}^{1 / 2} f\right\|_{L^{2}}^{2}
$$

and hence $\lambda_{g}>0$.

Now suppose condition 2 holds. We claim that for $\eta \in[0,1]$, the kernel of $\mathcal{P}_{\eta}$ is trivial. Since $\mathcal{P}_{\eta}$ has index 0 , this implies $\mathcal{P}_{\eta}$ is an isomorphism.

Suppose, to produce a contradiction, that $u$ is a nontrivial solution. From Lemma 3.3 we have $u \in W_{\delta^{\prime}}^{k, p}$ for any $\delta^{\prime} \in(2-n, 0)$. From Sobolev embedding we have $u \in W_{\delta^{\prime}}^{1,2}(M)$. Taking $\delta^{\prime}<1-n / 2$, we can integrate by parts to obtain

$$
0=\int_{M}-a u \Delta u+\eta R u^{2} d V=\int_{M} a|\nabla u|^{2}+\eta R u^{2} d V+\eta \int_{\partial M} \frac{a}{\kappa} h u^{2} d A .
$$

Since $\int_{M} a|\nabla u|^{2} d V \geq 0$, and since $\eta \in[0,1]$ we see

$$
0 \geq \eta\left[\int_{M} a|\nabla u|^{2}+R u^{2} d V+\int_{\partial M} \frac{a}{\kappa} h u^{2} d A\right]
$$


Since $W_{\delta^{\prime}}^{1,2}(M)$ is continuously embedded in $L^{2^{*}}(M)$ we conclude that $Q_{g}(u) \leq 0$. Since $Q_{g}$ is continuous on $W^{k, p} \delta^{\prime}$, and since $C_{\mathrm{c}}^{\infty}$ is dense in $W_{\delta^{\prime}}^{k, p}$ we find $\lambda_{g} \leq 0$, a contradiction.

Now suppose condition 3 holds. For each $\eta \in[0,1]$, let $v_{\eta}$ be the unique solution in $W_{\delta}^{k, p}$ of

$$
\mathcal{P}_{\eta} v_{\eta}=-\eta(R / a, h / \kappa)
$$

Letting $\phi_{\eta}=1+v_{\eta}$ we see

$$
\begin{aligned}
-\Delta \phi_{\eta}+\frac{\eta}{a} R \phi_{\eta} & =0 \\
\partial_{\nu} \phi_{\eta}+\frac{\eta}{\kappa} h \phi_{\eta} & =0 .
\end{aligned}
$$

To show $\phi_{\eta}>0$ for all $\eta \in[0,1]$, we follow [CaB81]. Let $I=\left\{\eta \in[0,1]: \phi_{\eta}>0\right\}$. Since $v_{0}=0$, we have $I$ is nonempty. Moreover, the set $\left\{v \in C_{\delta}^{0}: v>-1\right\}$ is open in $C_{\delta}^{0}$. Since the map taking $\eta$ to $v_{\eta} \in C_{\delta}^{0}$ is continuous, $I$ is open. It suffices to show that $I$ is closed. Suppose $\eta_{0} \in \bar{I}$. Then $\phi_{\eta_{0}} \geq 0$. Since $\phi_{\eta}$ solves (19), and since $\phi_{\eta_{0}}$ tends to 1 at infinity, Lemma 3.4 then implies $\phi_{\eta_{0}}>0$. Hence $\eta_{0} \in I$ and $I$ is closed.

Letting $\phi=\phi_{1}$ we have shown $\phi>0$. Since $\phi$ solves (19) with $\eta=1$ it follows that $\left(M, \phi^{2 \kappa} g\right)$ is scalar flat and has a minimal surface boundary. Moreover, since $v \in W_{\delta}^{k, p}$ we see $\left(M, \phi^{2 \kappa} g\right)$ is also of class $W_{\delta}^{k, p}$.

Remark. In the context of asymptotically Euclidean manifolds without boundary, Theorem 2.1 of [CaB81] claims that one can make a conformal change to a scalar flat asymptotically Euclidean metric if and only if

$$
\int_{M} a|\nabla f|^{2}+R f^{2} d V>0 \text { for all } f \in C_{\mathrm{c}}^{\infty}(M), f \not \equiv 0 .
$$

The proof of Theorem 2.1 in [CaB81] has a mistake, and the condition (20) is too weak. A similar claim and error appears in [CBIY00]. We see from Proposition 4.1 that the correct condition is

$$
\inf _{f \in C_{\mathrm{c}}^{\infty}(M), f \neq 0} \frac{\int_{M} a|\nabla f|^{2}+R f^{2} d V}{\|f\|_{L^{2^{*}}}^{2}}>0 .
$$

Corollary 4.2. Suppose $\left(M, g^{\prime}\right)$ is asymptotically Euclidean of class $W_{\delta}^{k, p}, k \geq 2, k>n / p$, and $2-n<\delta<0$. If $\lambda_{g^{\prime}}>0$, then there exists a conformal factor $\phi>0$ such that $1-\phi \in W_{\delta}^{k, p}$ and such that $(M, g)=\left(M, \phi^{2 \kappa} g^{\prime}\right)$ is scalar flat, has negative boundary mean curvature, and satisfies $\lambda_{g}>0$.

Proof: Since $\lambda_{g^{\prime}}>0$, from Proposition 4.1 we can assume without loss of generality that $\left(M, g^{\prime}\right)$ satisfies $R^{\prime}=0$ and $h^{\prime}=0$. 
Let $v_{\epsilon} \in W_{\delta}^{k, p}(M)$ be the unique solution of

$$
\begin{aligned}
-\Delta_{g^{\prime}} v_{\epsilon} & =0 \\
\partial_{\nu^{\prime}} v_{\epsilon} & =-\epsilon .
\end{aligned}
$$

Since $k>n / p, v_{\epsilon}$ depends continuously in $C_{\delta}^{0}$ on $\epsilon$. Since $v_{0}=0$, we have $v_{\epsilon}>-1$ for $\epsilon$ sufficiently small. Fixing one such $\epsilon>0$ we have $\phi=1+v_{\epsilon}>0$. Letting $g=\phi^{2 \kappa} g^{\prime}$ we see that $R=0$ and $h=-\epsilon \kappa \phi^{-\kappa-1}<0$. Proposition 4.1 shows that $\lambda_{g}$ is a conformal invariant and hence $\lambda_{g}>0$ also.

Theorem 4.3. Suppose $(M, g)$ is asymptotically Euclidean of class $W_{\delta}^{k, p}, k \geq 2, k>n / p$, and $2-n<\delta<0$. Suppose also that $\lambda_{g}>0, R=0$, and $h \leq 0$. If $\sigma \in W_{\delta-1}^{k-1, p}$ is a transverse traceless tensor on $M$ such that $(n-1) h \leq \sigma(\nu, \nu) \leq 0$ on $\partial M$, then there exists a conformal factor $\phi$ solving (17). Moreover, setting $\hat{g}=\phi^{2 \kappa} g$ and $\hat{K}=\phi^{-2} \sigma$, we have that $(M, \hat{g})$ is asymptotically Euclidean of class $W_{\delta}^{k, p}, \hat{K} \in W_{\delta-1}^{k-1, p},(M, \hat{g}, \hat{K})$ solves the Einstein constraint equations with apparent horizon boundary condition, and $\partial M$ is a marginally trapped surface.

Proof: Setting $\phi=1+v$ and $\sigma^{\prime}=\frac{2}{a} \sigma(\nu, \nu)$, the Lichnerowicz equation reduces to solving

$$
\begin{aligned}
-\Delta v & =\frac{1}{a}|\sigma|^{2}(1+v)^{-3-2 \kappa} \\
\partial_{\nu} v & =-\frac{1}{\kappa} h(1+v)+\sigma^{\prime}(1+v)^{-1-\kappa} \quad \text { on } \partial M
\end{aligned}
$$

with the constraint $v>-1$. We solve this by means of Proposition 3.5. Since $\frac{a}{2 \kappa}=n-1$, and since $(n-1) h \leq \sigma(\nu, \nu)$, we conclude $-\frac{1}{\kappa} h+\sigma^{\prime} \geq 0$. Therefore $v_{-}=0$ is a subsolution of (21). To find a supersolution, we solve for each $\eta \in[0,1]$

$$
\begin{aligned}
-\Delta v_{\eta} & =\frac{1}{a}|\sigma|^{2} \\
\partial_{\nu} v_{\eta}+\frac{\eta}{\kappa} h v_{\eta} & =-\frac{\eta}{\kappa} h .
\end{aligned}
$$

The solution exists since $\lambda_{g}>0$. We claim moreover that $\phi_{\eta}=1+v_{\eta}>0$. Let $I=\left\{\eta \in[0,1]: \phi_{\eta}>0\right\}$. Arguing as in Proposition 4.1, using the fact that $|\sigma|^{2} \geq 0$, we see $I$ is open and nonempty. Suppose $\eta_{0} \in \bar{I}$. Then $\phi_{\eta_{0}} \geq 0$. Since $\phi_{\eta}$ satisfies

$$
\begin{aligned}
-\Delta \phi_{\eta} & \geq 0 \\
\partial_{\nu} p h i_{\eta}+\frac{\eta}{\kappa} h \phi_{\eta} & =0,
\end{aligned}
$$

and since $\phi_{\eta_{0}}$ tends to 1 at infinity, Lemma 3.4 then implies $\phi_{\eta_{0}}>0$. Hence $\eta_{0} \in I$ and $I$ is closed. Let $v_{+}=v_{1}$. We have proved $1+v_{+}>0$. But then, since

$$
\begin{aligned}
-\Delta v_{+} & =\frac{1}{a}|\sigma|^{2} \\
\partial_{\nu} v_{+} & =-\frac{1}{\kappa} h\left(1+v_{+}\right),
\end{aligned}
$$


and since $-h \geq 0,1+v_{+} \geq 0$ and $|\sigma|^{2} \geq 0$, we conclude that $v_{+} \geq 0$. Now

$$
-\Delta v_{+}=\frac{1}{a}|\sigma|^{2} \geq \frac{1}{a}|\sigma|^{2}\left(1+v_{+}\right)^{-3-2 \kappa},
$$

and since $\sigma^{\prime} \leq 0$ we have

$$
\partial_{\nu} v_{+}=-\frac{1}{\kappa} h\left(1+v_{+}\right) \geq-\frac{1}{\kappa} h\left(1+v_{+}\right)+\sigma^{\prime}\left(1+v_{+}\right)^{-1-\kappa} \quad \text { on } \partial M .
$$

So $v_{+}$is a nonnegative supersolution of (21).

Now $v_{-}, v_{+},(M, g)$, and the right hand sides of (21) all satisfy the hypotheses of Proposition 3.5. So there exists a nonnegative solution $v$ of (21) in $W_{\delta}^{k, p}$. Letting $\hat{g}=\phi^{2 \kappa} g$ and $\hat{K}=\phi^{-2} \sigma$, it follows that $(M, \hat{g}, \hat{K})$ solves the constraint equations with apparent horizon boundary condition. To see that the boundary is marginally trapped, we note that

$$
(n-1) \hat{h}=\hat{K}(\hat{\nu}, \hat{\nu})=\phi^{-2-2 \kappa} \sigma(\nu, \nu) \leq 0 .
$$

Since $\hat{\theta}_{-}=\hat{\theta}_{+}+2(n-1) \hat{h}$, we conclude $\hat{\theta}_{-} \leq \hat{\theta}_{+}=0$, and $\partial M$ is marginally trapped.

The previous arguments and theorems can all be easily modified for manifolds without boundary by omitting boundary conditions and all references to the boundary of the manifold. Hence we also have the following low regularity construction for manifolds without boundary.

Theorem 4.4. Suppose $(M, g)$ is an asymptotically Euclidean manifold without boundary of class $W_{\delta}^{k, p}, k \geq 2, k>n / p, 2-n<\delta<0$, and suppose $\sigma \in W_{\delta-1}^{k-1, p}$. If $(M, g)$ satisfies $\lambda_{g}>0$, then there exists a conformal factor $\phi$ solving (17). Moreover, $(M, \hat{g})=\left(M, \phi^{2 \kappa} g\right)$ is asymptotically Euclidean of class $W_{\delta}^{k, p}, \hat{K}=\phi^{-2} \sigma \in W_{\delta-1}^{k-1, p}$, and $(M, \hat{g}, \hat{K})$ solves the Einstein constraint equations.

\section{Constructing Suitable Transverse Traceless Tensors}

We now prove the existence of a class of data satisfying the hypothesis of Theorem 4.3. It is easy to construct asymptotically Euclidean manifolds satisfying $\lambda_{g}>0$. From the proof of Proposition 4.1, it is clear that every manifold with $R \geq 0$ and $h \geq 0$ satisfies $\lambda_{g}>0$. Let $(M, g, K)$ be an asymptotically Euclidean manifold without boundary satisfying $R \geq 0$ (for example any maximal solution of the vacuum constraint equations). Let $v$ be the Greens function for the operator $-a \Delta+R$ with pole at $x \in M$, and let $\phi=1+v$. Since $R \geq 0$, we have $v \geq 0$ and $\phi>0$. Setting $\tilde{g}=\phi^{2 k} g$ we see that $(M-\{x\}, \tilde{g})$ satisfies $\tilde{R} \geq 0$. Moreover, if $B_{r}$ is a geodesic ball (with respect to $g$ ) about $x$ with radius $r$, one readily verifies from the asymptotic behaviour $\phi \approx r^{2-n}$ near $x$ that $\tilde{h}>0$ 
for $r$ sufficiently small. So $\left(M-B_{r}, \tilde{g}\right)$ satisfies $\lambda_{\tilde{g}}>0$. In the same way, given an asymptotically Euclidean manifold with boundary satisfying $R \geq 0$ and $h \geq 0$ we can perform the previous construction using the Greens function for $-a \Delta+R$ corresponding to the boundary condition $\partial v+h v=0$ to add another boundary component. Continuing iteratively, we can add as many boundary components as we like.

On the other hand, to provide a suitable tensor $\sigma$, we must do more work. The requirements on $\sigma$ are that it be trace-free, divergence-free, and satisfy $(n-1) h \leq \sigma(\nu, \nu) \leq 0$ on $\partial M$. We construct $\sigma$ using a boundary value problem for the vector Laplacian.

Let $\mathbb{L}$ denote the conformal Killing operator, so $\mathbb{L} X=\frac{1}{2} \mathcal{L}_{X} g-\frac{1}{n}(\operatorname{div} X) g$. Then the vector Laplacian $\Delta_{\mathbb{L}}=\operatorname{div} \mathbb{L}$ is an elliptic operator on $M$, and the Neumann boundary operator $B$ corresponding to $\Delta_{\mathbb{L}}$ takes a vector field $X$ to the covector field $\mathbb{L} X(\nu, \cdot)$. We propose to solve the boundary value problem

$$
\begin{aligned}
\Delta_{\mathbb{L}} X & =0 \\
B X & =\omega \quad \text { on } \partial M,
\end{aligned}
$$

where $\omega$ is a covector field over $\partial M$. If we can do this, then letting $\sigma=\mathbb{L} X$ it follows that $\sigma$ is trace and divergence free. Moreover, $\sigma(\nu, \nu)=\omega(\nu)$ on $\partial M$, so taking $\omega$ such that $(n-1) h \leq \omega(\nu) \leq 0$ ensure $(n-1) h \leq \sigma(\nu, \nu) \leq 0$.

If $(M, g)$ is asymptotically Euclidean of class $W_{\rho}^{k, p}$ with $k \geq 2$ and $k>n / p$, then $\Delta_{\mathbb{L}}$ and $B$ act continuously as maps from $W_{\delta}^{k, p}(M)$ to $W_{\delta-2}^{k-2, p}(M)$ and $W^{k-1-\frac{1}{p}, p}(\partial M)$ respectively. We show (24) is well posed by proving $\mathcal{P}_{\mathbb{L}}=\left(\Delta_{\mathbb{L}}, B\right)$ is an isomorphism if $2-n<\delta<0$ and either $k>n / p+1$ or $k>n / p$ and $(M, g)$ has no nontrivial conformal Killing fields vanishing at infinity.

The method of proof is well established [Mc79] [CBC81]. The first step is to obtain a coercivity estimate (equation (29) of Proposition 5.2 below) that implies $\mathcal{P}_{\mathbb{L}}$ is semiFredholm. The second step is to explicitly compute the index and dimension of the kernel of $\mathcal{P}_{\mathbb{L}}$, which we do in Proposition 5.3.

In fact, for smooth metrics it follows from [LM85] that $\mathcal{P}_{\mathbb{L}}$ is Fredholm. So our principal concern is to show that the coercivity estimate holds for low regularity metrics.

We start with an priori estimate for $\Delta_{\mathbb{L}}$ on a compact manifold $K$. We assume that the boundary of $K$ is partitioned into two pieces, $\partial K_{1}$ and $\partial K_{2}$, each the union of components of $\partial K$ and either possibly empty. We then have an estimate for $\Delta_{\mathbb{L}}$ in terms of a Neumann condition on $K_{1}$ and a Dirichlet condition on $K_{2}$.

Proposition 5.1. Suppose $(K, g)$ is a compact manifold with boundary of class $W^{k, p}$ with 
$k>n / p$ and $k \geq 2$. If $X \in W^{k, p}(K)$, then

$$
\begin{gathered}
\|X\|_{W^{k, p}(K)} \lesssim\left\|\Delta_{\mathbb{L}} X\right\|_{W^{k-2, p}(K)}+\left.\|B X\|\right|_{W^{k-1-\frac{1}{p}, p}\left(\partial K_{1}\right)}+ \\
+\|X\|_{W^{k-\frac{1}{p}, p}\left(\partial K_{2}\right)}+\|X\|_{L^{p}(K)} .
\end{gathered}
$$

Proof: The estimate follows from interior and boundary estimates together with a partition of unity argument. We explicitly prove the local estimate near $\partial K_{1}$, the other estimates being similar and easier. In local coordinates near some fixed $x \in \partial K$ we may assume the boundary is flat, $x=0$, and $g_{i j}(0)=\delta_{i j}$. In these coordinates,

$$
\Delta_{\mathbb{L}} X_{j}=\sum_{i, j,|\alpha| \leq 2} c_{j}^{i, \alpha} \partial_{\alpha} X_{i}
$$

where $c_{j}^{i, \alpha} \in W_{\mathrm{loc}}^{k-2+|\alpha|, p}$.

Suppose first that $X \in W^{k, p}\left(B_{r}^{+}\right)$with support contained in $B_{r / 2}^{+}$, where $r$ is a small number to be specified later. Let $\bar{\Delta}_{\mathbb{L}}$ and $\bar{B}$ denote the constant coefficient operators given by the principal symbols of $\Delta_{\mathbb{L}}$ and $B$ at 0 ; these are in fact the vector Laplacian and Neumann boundary operator computed with respect to the Euclidean metric on the half space. It is easy to verify that these satisfy the so-called Lopatinski-Shapiro or covering conditions of [ADN64] and hence we have

$$
\|X\|_{W^{k, p}\left(B_{r}^{+}\right)} \leq\left\|\bar{\Delta}_{\mathbb{L}} X\right\|_{W^{k-2, p}\left(B_{r}^{+}\right)}+\|\bar{B} X\|_{W^{k-1-\frac{1}{p}, p}\left(D_{r}\right)}+\|X\|_{W^{k-2, p}\left(B_{r}^{+}\right)},
$$

where $D_{r}$ is the flat portion of $\partial B_{r}^{+}$. In local coordinates

$$
\left(\bar{\Delta}_{\mathbb{L}} X\right)_{j}=\left(\Delta_{\mathbb{L}} X\right)_{j}+\sum_{|\alpha|=2}\left(\bar{c}_{j}^{i, \alpha}-c_{j}^{i, \alpha}\right) \partial_{\alpha} X_{i}+\sum_{|\alpha|<2} c_{j}^{i, \alpha} \partial_{\alpha} X_{i}
$$

We wish to estimate each of these terms in $W^{k-2, p}\left(B_{r}^{+}\right)$, and we must proceed carefully since a naive application of the multiplication rule from Lemma 2.1 will introduce unwanted

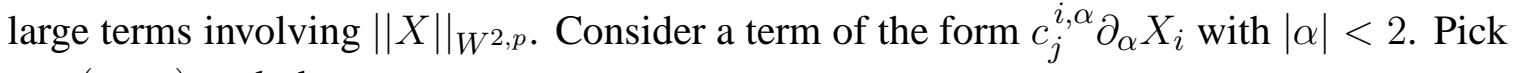
$q \in(p, \infty)$ such that

$$
\frac{1}{p}-\frac{1}{n}<\frac{1}{q}<\frac{k-1}{n} .
$$

Then applying Lemma 2.1 we have

$$
\left\|c_{j}^{i, \alpha} \partial_{\alpha} X_{i}\right\|_{W^{k-2, p}\left(B_{r}^{+}\right)} \lesssim\left\|X_{i}\right\|_{W^{k-1, q}\left(B_{r}^{+}\right)}
$$

and, arguing as in Proposition 3.5, we obtain from interpolation and Sobolev embedding

$$
\left\|c_{j}^{i, \alpha} \partial_{\alpha} X_{i}\right\|_{W^{k-2, p}\left(B_{r}^{+}\right)} \lesssim C(\epsilon)\|X\|_{L^{p}\left(B_{r}^{+}\right)}+\epsilon\|X\|_{W^{k, p}\left(B_{r}^{+}\right)} .
$$


The terms involving $\left(\bar{c}_{j}^{i, \alpha}-c_{j}^{i, \alpha}\right) \partial_{\alpha} X_{i}$ are estimated similarly, and we have

$$
\left(\bar{c}_{j}^{i, \alpha}-c_{j}^{i, \alpha}\right) \partial_{\alpha} X_{i} \lesssim C(\epsilon)\|X\|_{L^{p}\left(B_{r}^{+}\right)}+\left(\left\|\bar{c}_{j}^{i, \alpha}-c_{j}^{i, \alpha}\right\|_{L^{\infty}}+\epsilon\right)\left\|X_{i}\right\|_{W^{k, p}\left(B_{r}^{+}\right)} .
$$

Since $\left\|\bar{c}_{j}^{i, \alpha}-c_{j}^{i, \alpha}\right\|_{L^{\infty}}$ can be made as small as we please by taking $r$ small enough we conclude

$$
\left\|\bar{\Delta}_{\mathbb{L}} X\right\|_{L^{p}\left(B_{r}^{+}\right)} \lesssim\left\|\Delta_{\mathbb{L}} X\right\|_{L^{p}\left(B_{r}^{+}\right)}+C(\epsilon)\|X\|_{L^{p}\left(B_{r}^{+}\right)}+\epsilon\|X\|_{W^{2 p,\left(B_{r}^{+}\right)}} .
$$

A similar argument with the boundary operator leads us to

$$
\begin{aligned}
\|\bar{B} X\|_{W^{k-1-\frac{1}{p}, p}\left(D_{r}\right)} \lesssim & \left\|(B X)_{j}\right\|_{W^{k-1-\frac{1}{p}, p}\left(D_{r}\right)}+ \\
& C(\epsilon)\|X\|_{W^{k-1, p}\left(B_{r}^{+}\right)}+\epsilon\|X\|_{W^{k, p}\left(B_{r}^{+}\right)} .
\end{aligned}
$$

Combining (26), (27) and (28), taking $\epsilon$ sufficiently small, we conclude

$$
\|X\|_{W^{k, p}\left(B_{r}^{+}\right)} \lesssim\left\|\Delta_{\mathbb{L}} X\right\|_{W^{k-2, p}\left(B_{r}^{+}\right)}+\|B X\|_{W^{k-1-\frac{1}{p}, p}\left(D_{r}\right)}+\|X\|_{W^{k-1, p}\left(B_{r}^{+}\right)} .
$$

The estimate (25) for $X$ with arbitrary support is now achieved in a standard way with cutoff functions and a partition of unity argument.

With the a priori estimate for compact manifolds in hand, the following coercivity result is now standard [Ca79] [CBC81][Ba86]. We omit the proof for the sake of brevity.

Proposition 5.2. Suppose $(M, g)$ is asymptotically Euclidean of class $W_{\rho}^{k, p}$ with $k>n / p$ and $k \geq 2$. Then if $2-n<\delta<0, \delta^{\prime} \in \mathbb{R}$, and $X \in W_{\delta}^{k, p}(M)$ we have

$$
\|X\|_{W_{\delta}^{k, p}(M)} \lesssim\left\|\Delta_{\mathbb{L}} X\right\|_{W_{\delta-2}^{k-2, p}(M)}+\|B X\|_{W^{k-1-\frac{1}{p}, p}(\partial M)}+\|X\|_{L_{\delta^{\prime}}^{p}(M)},
$$

noting that the inequality is trivial if $\|X\|_{L_{\delta^{\prime}}^{p}(M)}=\infty$.

Let $\mathcal{P}_{\delta}^{k, p}$ denote $\mathcal{P}_{\mathbb{L}}$ acting as a map from $W_{\delta}^{k, p}(M)$ to $W_{\delta-2}^{k-2, p}(M) \times W^{k-1-\frac{1}{p}, p}(\partial M)$.

From estimate (29) it follows immediately [Sc02] that $\mathcal{P}_{\delta}^{k, p}$ is semi-Fredholm under the assumptions on $k, p$, and $\delta$ of Proposition 5.2. We now show $\mathcal{P}_{\delta}^{k, p}$ is Fredholm with index 0 .

Proposition 5.3. Suppose $(M, g)$ is asymptotically Euclidean of class $W_{\rho}^{k, p}$ with $k>n / p$, $k \geq 2$, and suppose $2-n<\delta<0$. Then $\mathcal{P}_{\delta}^{k, p}$ is Fredholm of index 0. Moreover, it is an isomorphism if and only if $(M, g)$ possesses no nontrivial conformal Killing fields in $W_{\delta}^{k, p}(M)$.

Proof: We first suppose $(M, g)$ is asymptotically flat of class $C_{\rho}^{\infty}$; the desired result for rough metrics will follow from an index theory argument. 
It is enough to prove that $\mathcal{P}_{\delta}^{2,2}$ is invertible. Indeed, from elliptic regularity we know that the the kernels of $\mathcal{P}_{\delta}^{k, p}$ and $\mathcal{P}_{\delta}^{2,2}$ agree. And if $\mathcal{P}_{\delta}^{2,2}$ is surjective, then the image of $\mathcal{P}_{\delta}^{2,2}$ contains $C_{\mathrm{c}}^{\infty}(M) \times C^{\infty}(\partial M)$. Again from elliptic regularity we have that the image of $\mathcal{P}_{\delta}^{k, p}$ also contains $C_{\mathrm{c}}^{\infty}(M) \times C^{\infty}(\partial M)$ and since the image of $\mathcal{P}_{\delta}^{k, p}$ is closed, $\mathcal{P}_{\delta}^{k, p}$ is surjective.

We restrict our attention now to $\mathcal{P}=\mathcal{P}_{\delta}^{2,2}$. To show $\mathcal{P}$ is injective, we prove that any element of the kernel of $\mathcal{P}$ is a conformal killing field. Since $g$ is smooth, it follows from [CO81] (see also Section 6) that there are no nontrivial conformal Killing fields in $W_{\delta}^{2,2}$, and hence the kernel of $\mathcal{P}$ is trivial. Suppose $u \in \operatorname{ker} \mathcal{P}$. We would like to integrate by parts to deduce that

$$
0=-\int_{M}<\Delta_{\mathbb{L}} u, u>d V=\int_{M}<\mathbb{L} u, \mathbb{L} u>d V
$$

and hence $u$ is a conformal Killing field. This computation is only valid if $\delta \leq 1-\frac{n}{2}$. It was shown in [CO81] (in the case of manifolds without boundary) that if $u \in \operatorname{ker} \mathcal{P}$, then $u \in$ $W_{\delta^{\prime}}^{2,2}$ for every $\delta^{\prime} \in(2-n, 0)$. Their proof also works if $\mathcal{P} u$ is only compactly supported, and a simple hole filling argument then implies the same result holds for manifolds with boundary. So $\mathcal{P}$ is injective.

To show $\mathcal{P}$ is surjective, it is enough to show that the adjoint $\mathcal{P}^{*}$ is injective. The dual space of $L_{\delta-2}^{2}(M) \times H^{1 / 2}(\partial M)$ is $L_{2-n-\delta}^{2}(M) \times H^{-1 / 2}(\partial M)$. From elliptic regularity [Hö85] and rescaled interior estimates we know that if $\mathcal{P}^{*}(f, h)=0$, then in fact $f$ and $h$ are smooth and $f \in H_{2-n-\delta}^{2}(M)$. Now if $\phi$ is smooth and compactly supported in each end of $M$ we have from integrating by parts

$$
\begin{aligned}
0 & =\langle\mathcal{P}(f, h), \phi\rangle \\
& =\int_{M}<\Delta_{\mathbb{L}} f, \phi>d V+\int_{\partial M} \mathbb{L} \phi(\nu, f+h)-\mathbb{L} f(\nu, \phi) d A .
\end{aligned}
$$

We obtain immediately $\Delta_{\mathbb{L}} f=0$ in $M$. Moreover, one can readily show that if $\omega$ is a smooth 1 -form on $\partial M$ and $\psi$ is a smooth function on $\partial M$, then there exists a $\phi \in C_{\mathrm{c}}^{\infty}$ such that $\phi=\psi$ and $\mathbb{L} \phi=\omega$ on $\partial M$. It follows that $B f=0$ and $h=0$. Since $\Delta_{\mathbb{L}} f=0$ and $B f=0$, we have $f=0$. Hence $\mathcal{P}^{*}$ is injective and therefore $\mathcal{P}$ is an isomorphism.

We return to the case where $g$ is not smooth but only in $W_{\rho}^{k, p}(M)$ with $k>n / p$ and $k \geq 2$. To show $\mathcal{P}_{\delta}^{k, p}$ is Fredholm of index 0, it is enough to show its index is 0 . Since $g$ can be approximated with smooth metrics $g_{k}$, and since each $\mathcal{P}_{\delta, g_{k}}^{k, p}$ has index 0 , so does the limit $\mathcal{P}_{\delta}^{k, p}$. To show that the kernel of $\mathcal{P}_{\delta}^{k, p}$ consists of conformal Killing fields, we integrate by parts again using the fact that elements in the kernel decay sufficiently fast at infinity.

Proposition 5.3 reduces the question of whether or not $\mathcal{P}_{\mathbb{L}}$ is an isomorphism to the existence of nontrivial conformal Killing fields $X$ vanishing at infinity with $\mathbb{L} X(\nu, \cdot)=0$ on $\partial M$. 
In [CO81] it was proved that if $(M, g)$ is asymptotically Euclidean of class $W_{\rho}^{k, p}$ with $k>n / p+3$ that there are no nontrivial conformal Killing fields in $W_{\delta}^{k, p}$ for $\delta<0$. Hence the boundary value problem (24) is well posed if the metric has this high degree of regularity. It has been subsequently claimed [CBIY00] that no nontrivial conformal Killing fields exist with the metric as irregular as $k>n / p+2$, but no proof exists in the literature. The following section contains a proof that there are no such vector fields for metrics as irregular as $k>n / p+1$ and thereby establishes

Theorem 5.4. Suppose $(M, g)$ is asymptotically Euclidean of class $W_{\rho}^{k, p}$ with $k \geq 2$ and suppose $2-n<\delta<0$. If either $k>n / p+1$ or $k>n / p$ and $(M, g)$ has no nontrivial conformal Killing fields in $W_{\delta}^{k, p}(M)$, then there exists a unique solution $X \in W_{\delta}^{k, p}(M)$ of (24).

\section{Non-Existence of Conformal Killing Fields Vanishing at Infinity}

We break the problem of showing conformal Killing fields vanishing at infinity are trivial into two pieces as was done in the high regularity proof of [CO81]. We first show that if a conformal Killing field exists, it must be identically zero in a neighbourhood of each end. We then show that the zero set can be extended to encompass the whole manifold. For both parts of the argument, we use a blowup method to construct a conformal Killing field on a subset of $\mathbb{R}^{n}$ and analyze properties of the resulting limit field.

In this section we use the notation $e_{i}$ for a standard basis element of $\mathbb{R}^{n}$ or $\mathbb{R}^{n+1}$. Recall [KP88] that a basis for the conformal Killing fields on $\mathbb{R}^{n}$ with the Euclidean metric is comprised of the generators of the translations $\left\{e_{i}\right\}_{i=1}^{n}$, the rotations $\left\{x_{i} e_{j}-x_{j} e_{i}\right\}_{1 \leq i<j \leq n}$ and the spherical dilations $\left\{D_{e_{k}}\right\}_{k=1}^{n+1}$. Our notation for the dilations is as follows. Any constant vector $V$ in $R^{n+1}$ gives rise to a function $x \mapsto<V, x>$ on the sphere. The field $D_{V}$ is the pushforward under stereographic projection of the gradient of this function. We note that $D_{V_{1}}+D_{V_{2}}=D_{V_{1}+V_{2}}$. Moreover, in local coordinates we have

$$
D_{e_{1}}=\left(\frac{1}{2}\left(1-x_{1}^{2}+x_{2}^{2}+\cdots+x_{n}^{2}\right),-x_{1} x_{2}, \cdots,-x_{1} x_{n}\right) .
$$

The dilations $D_{e_{j}}$ for $1<j \leq n$ have similar coordinate expressions being the pushforward of $D_{e_{1}}$ under a rotation. Finally, $D_{e_{n+1}}$ has the coordinate expression

$$
D_{e_{n+1}}=\left(x_{1}, \cdots, x_{n}\right) \text {. }
$$

The following two lemmas provide properties of conformal Killing fields on subsets $\mathbb{R}^{n}$. The first one can also be deduced from the analysis in [CO81], but we include it here for completeness. 
Lemma 6.1. Suppose $X$ is a conformal Killing field on the external domain $E_{1}$ with the Euclidean metric. Suppose moreover that $X \in L_{\delta}^{p}\left(E_{1}\right)$ for some $p \geq 1$ and $\delta<0$. Then $X$ vanishes identically.

Proof: The basis of conformal Killing fields on $\mathbb{R}^{n}$ restricts to a basis of conformal Killing fields on $E_{1}$. If $X$ is a sum of such vectors, its coefficients are polynomials. But no non-zero polynomial is in $L_{\delta}^{p}\left(E_{1}\right)$, since $\delta<0$. Hence $X=0$.

Lemma 6.2. Suppose $X$ is a nontrivial conformal Killing field on $B_{1}\left(\mathbb{R}^{n}\right)$ with the Euclidean metric that satisfies $X(0)=0$ and $\nabla X(0)=0$. Then $X(x) \neq 0$ if $x \neq 0$.

Proof: By a conformal change of metric, it is enough to show the same is true on $\mathbb{R}^{n}$. A routine computation shows that the subspace of conformal Killing vector fields in $\mathbb{R}^{n}$ that vanish at 0 is spanned by the rotations, the dilation $D_{e_{n+1}}$, and the vectors

$$
2 D_{e_{i}}-e_{i} \quad i=1 \cdots n \text {. }
$$

The coefficients of the vectors (32) are homogeneous polynomials of degree 2 and hence their derivatives at the origin vanish. On the other hand, a vector in the span of $D_{e_{n+1}}$ and the rotations has linear coefficients and vanishes identically if its gradient at the origin vanishes. So if $X(0)=0$ and $\nabla X(0)=0$, then $X$ is in the span of of the vectors (32). We claim a nontrivial such vector vanishes only at the origin. Indeed,

$$
\sum_{j=1}^{n} v^{j}\left(2 D_{e_{j}}-e_{j}\right)=2 D_{V}-V
$$

where $V=\sum_{j=1}^{n} v^{j} e_{j}$. If $V$ is not zero, then by performing a rotation and constant scaling we can push $2 D_{V}-V$ forward to $2 D_{e_{1}}-e_{1}$. From the explicit expression (31) we observe that $2 D_{e_{1}}-e_{1}$ vanishes only at 0 .

We say a vector field $X$ vanishes in a neighbourhood of infinity if for each end there exists a radius $R$ such that in end coordinates $X \equiv 0$ in the exterior region $E_{R}$. The following lemma shows under weak hypotheses on the metric that a conformal Killing field vanishing at infinity also vanishes in a neighbourhood of infinity.

Lemma 6.3. Suppose $(M, g)$ is asymptotically Euclidean of class $W_{\rho}^{2, p}$ with $p>n / 2$. Suppose $X$ is a conformal Killing field in $W_{\delta}^{2, p}$ with $\delta<0$. Then $X$ vanishes in a neighbourhood of infinity.

Proof: We work in end coordinates and construct a sequence of metrics $\left\{g_{k}\right\}_{k=1}^{\infty}$ on the exterior region $E_{1}$ by letting $g_{k}(x)=g\left(2^{k} x\right)$. Since $\left\|g_{k}-\bar{g}\right\|_{W_{\rho}^{2, p}\left(E_{1}\right)} \lesssim 2^{\rho k}\|g-\bar{g}\|_{W_{\rho}^{2, p}\left(E_{2^{k}}\right)}$ 
we conclude $\left\|g_{k}-\bar{g}\right\|_{W_{\rho}^{2, p}\left(E_{1}\right)} \rightarrow 0$. It follows that the associated maps $\Delta_{\mathbb{L}}^{k}$ and $\mathbb{L}^{k}$ acting on $W_{\delta}^{2, p}\left(E_{1}\right)$ converge as operators to $\bar{\Delta}_{\mathbb{L}}$ and $\overline{\mathbb{L}}$.

Suppose, to produce a contradiction, that $X$ is not identically 0 outside any exterior region $E_{R}$. Let $\hat{X}_{k}$ be the vector field on $E_{1}$ given by $\hat{X}_{k}(x)=X\left(2^{k} x\right)$ and let $X_{k}=\hat{X}_{k} /\left\|\hat{X}_{k}\right\|_{W_{\delta}^{2, p}}$. Fix $\delta^{\prime}$ with $\delta^{\prime} \in(\delta, 0)$. Then from the $W_{\delta}^{2, p}$ boundedness of the sequence $\left\{X_{k}\right\}_{k=1}^{\infty}$ it follows (after reducing to a subsequence) that the vectors $X_{k}$ converge strongly in $W_{\delta^{\prime}}^{1, p}$ to some $X_{0}$. We can assume without loss of generality that $\delta>2-n$ and hence we can apply Proposition 5.2 to the Euclidean metric to obtain

$$
\begin{aligned}
\left\|X_{k_{1}}-X_{k_{2}}\right\|_{W_{\delta}^{2, p}} \lesssim\left\|\bar{\Delta}_{\mathbb{L}}-\Delta_{\mathbb{L}}^{k_{1}}\right\|_{W_{\delta}^{2, p}}+\left\|\bar{\Delta}_{\mathbb{L}}-\Delta_{\mathbb{L}}^{k_{2}}\right\|_{W_{\delta}^{2, p}}+ \\
+\left\|\overline{\mathbb{L}}-\mathbb{L}^{k_{1}}\right\|_{W_{\delta}^{2, p}}+\left\|\overline{\mathbb{L}}-\mathbb{L}^{k_{2}}\right\|_{W_{\delta}^{2, p}}+\left\|X_{k_{1}}-X_{k_{2}}\right\|_{L_{\delta^{\prime}}^{p}} .
\end{aligned}
$$

Here we have used the $W_{\delta}^{2, p}$ boundedness of the sequence $\left\{X_{k}\right\}_{k=1}^{\infty}$ together with the facts $\Delta_{\mathbb{L}}^{k} X_{k}=0$ and $\mathbb{L}^{k} X_{k}=0$. We conclude $\left\{X_{k}\right\}_{k=1}^{\infty}$ is Cauchy in $W_{\delta}^{2, p}\left(E_{1}\right)$ and hence $X_{k} \underset{W_{\delta}^{2, p}}{\longrightarrow} X_{0}$. Moreover, since $\mathbb{L}^{k} \underset{W_{\delta}^{2, p}}{\longrightarrow} \mathbb{L}$ and since $\mathbb{L}^{k} X_{k}=0$ we have $X_{0}$ is a conformal Killing field for $\bar{g}$ in $W_{\delta}^{2, p}\left(E_{1}\right)$. From Lemma 6.1 it follows that $X_{0}=0$. Hence $X_{k}$ converges in $W_{\delta}^{2, p}$ to 0 , which contradicts $\left\|X_{k}\right\|_{W_{\delta}^{2, p}}=1$ for each $k$.

Theorem 6.4. Suppose $(M, g)$ is asymptotically Euclidean of class $W_{\rho}^{2, p}$ with $p>n$. Then there exist no nontrivial conformal Killing fields in $W_{\delta}^{k, p}$ for any $\delta<0$.

Proof: From Lemma 6.3 we know that if $X \in W_{\delta}^{2, p}$ is a conformal Killing field then it vanishes on an open set. We claim $X^{-1}(0)=M$.

If the claim is not true, then there exists a point $x_{0}$ in the interior of $M$ and on the boundary of the interior of $X^{-1}(0)$. Working in local coordinates near $x$, we reduce to the situation where $g$ is a metric on the unit ball, $g_{i j}(0)=\delta_{i j}, X$ is a conformal Killing field for $g$ on $B_{1}$, and the origin is on the boundary of the interior of $X^{-1}(0)$. Since $p>n$ it follows that $X$ is in $C^{1}\left(B_{r}\right)$ and hence $X(0)=0$ and $\nabla X(0)=0$.

Let $\left\{r_{k}\right\}_{k=1}^{\infty}$ be a sequence of radii $r_{k}$ tending down to zero such that $X(x)=0$ for some $x$ with $|x|=r_{k} / 2$. We construct a sequence of metrics $\left\{g_{k}\right\}_{k=1}^{\infty}$ on the unit ball by taking $g_{k}(x)=g\left(x / r_{k}\right)$. Evidently, $g_{k} \underset{W^{2, p}}{\longrightarrow} \bar{g}$, and it follows that the associated maps $\Delta_{\mathbb{L}}^{k}$ and $\mathbb{L}^{k}$ converge to $\bar{\Delta}_{\mathbb{L}}$ and $\overline{\mathbb{L}}$ as operators on $W_{\delta}^{2, p}\left(B_{1}\right)$.

We construct vector fields $X_{k}$ on $B_{1}$ by setting $\hat{X}_{k}(x)=X\left(x / r_{k}\right)$ and letting $X_{k}=$ $\hat{X}_{k} /\left\|\hat{X}_{k}\right\|_{W^{2, p}}$ (since $X$ is not identically 0 on $B_{r_{k}}$ the normalization is possible). By our choice of radii, there exists a point $x_{k}$ with $\left|x_{k}\right|=1 / 2$ such that $X_{k}\left(x_{k}\right)=0$. From the 
$W^{2, p}$ boundedness of the sequence $\left\{X_{k}\right\}_{k=1}^{\infty}$, it follows (after taking a subsequence) that $X_{k}$ converges strongly in $W^{1, p}$ to some $X_{0} \in W^{2, p}$.

Arguing as in Lemma 6.3, replacing the use of Proposition 5.2 with Proposition 5.1, we conclude $X_{0}$ is a conformal killing field for $\bar{g}$ and $X_{k}$ converges in $W^{2, p}$ to $X_{0}$. From the resulting $C^{1}\left(B_{1}\right)$ convergence of $X_{k}$ to $X_{0}$ we know $X_{0}(0)=0$ and $\nabla X_{0}(0)=0$. The collection of points $x_{k}$ where $X_{k}$ vanishes has a cluster point $x$ with $|x|=1 / 2$, and hence $X_{0}(x)=0$. But Lemma 6.2 then implies $X_{0}=0$, which is impossible since $\left\|X_{k}\right\|_{W^{2, p}}=1$ for each $k$. Hence $X=0$ identically.

Remark. Even though Theorem 6.4 requires $W^{2, n+\epsilon}$ regularity, we know from Lemma 6.3 that a conformal Killing field that vanishes at infinity also vanishes in a neighbourhood of infinity, even if the metric only has $W^{2, \frac{n}{2}+\epsilon}$ regularity. Since Theorem 6.4 is a unique continuation argument, it is perhaps not surprising that it requires $g \in W_{\mathrm{loc}}^{2, n+\epsilon}$, as this is the minimal regularity that guarantees the principal coefficients of $\Delta_{\mathbb{L}}$ are Lipschitz continuous. It would be interesting to determine if Theorem 6.4 also holds in the low regularity case $W^{2, \frac{n}{2}+\epsilon}$.

Acknowledgements. I would like to thank D. Pollack, J. Isenberg, and S. Dain for helpful discussions and advice. I would also like to thank an anonymous referee for suggestions that improved the paper's style. This research was partially supported by NSF grant DMS0305048. 


\section{References}

[ADN64] S. Agmon, A. Douglis, and L. Nirenberg, Estimates near the boundary for solutions of elliptic partial differential equations satisfying general boundary conditions II, Comm. Pure Appl. Math. 17 (1964), 35-92.

[Ba86] R. Bartnik, The mass of an asymptotically flat manifold, Comm. Pure Appl. Math. 39 (1986), 661-693.

[BL63] D. Brill and R. Lindquist, Interaction energy in geometrostatics, Phys. Rev. (2) 131 (1963), 471-476.

[Ca79] M. Cantor, Some problems of global analysis on asymptotically simple manifolds, Compositio Math. 38 (1979), 3-35.

[CaB81] M. Cantor and D. Brill, The Laplacian on asymptotically flat manifolds and the specification of scalar curvature, Compositio Math. 43 (1981), no. 3, 317-330.

[CB03] Y. Choquet-Bruhat, Einstein constraints on compact n-dimensional manifolds, [gr-qc/0311029], 2003.

[CBC81] Y. Choquet-Bruhat and D. Christodoulou, Elliptic systems in $H_{s, \delta}$ spaces on manifolds which are Euclidean at infinity, Acta Math. 146 (1981), 129-150.

[CBIY00] Y. Choquet-Bruhat, J. Isenberg, and J. W. York, Jr, Einstein constraints on asymptotically Euclidean manifolds, Phys. Rev. D 61 (2000), 1-20.

[CBY80] Y. Choquet-Bruhat and J. W. York, Jr, The Cauchy problem, General Relativity and Gravitation (A. Held, ed.), Plenum, New York, 1980.

[Ck00] G. B. Cook, Initial data for numerical relativity, Living Rev. 5 (2000), [www.livingreviews.org/lrr-2000-5].

[Ck02] G. B. Cook, Corotating and irrotatinal binary black holes in quasi-circular orbits, Phys. Rev. D 65 (2002), 084003.

[CO81] D. Christodoulou and N. O'Murchadha, The boost problem in general relativity, Comm. Math. Phys. 80 (1981), 271-300.

[Da02] S. Dain, Initial data for black hole collisions, [gr-qc/0212039], 2002.

[Da03] - Trapped surfaces as boundaries for the constraint equations, [grqc/0308009], 2003.

[Ea98] D. M. Eardley, Black hole boundary conditions and coordinate conditions, Phys. Rev. D 57 (1998), no. 4, 2299-2304.

[Es92] J. F. Escobar, The Yambe problem on manifolds with boundary, J. Differential Geom. 35 (1992), 21-84.

[Hö85] L. Hörmander, Analysis of linear partial differential operators, vol. III, SpringerVerlag, Berlin, 1985. 
[Is95] J. Isenberg, Constant mean curvature solutions of the Einstein constraint equations on closed manifolds, Classical Quantum Gravity 12 (1995), 2249-2274.

[KP88] R. S. Kulkarni and U. Pinkall (eds.), Conformal geometry, Friedr. Vieweg \& Sohn, Weisbaden, 1988.

[KR02] S. Klainerman and I. Rodnianski, Rough solutions of the Einstein vacuum equations, C. R. Acad. Sci. Paris Sér. I Math. 334 (2002), 125-130.

[Li44] A. Lichernowicz, Sur l'intégration des équations d'Einstein, J. Math. Pures Appl. 23 (1944), 26-63.

[LM85] R. B. Lockhart and R. C. McOwen, Elliptic differential operators on noncompact manifolds, Ann. Scuola Norm. Sup. Pisa Cl. Sci. 12 (1985), no. 4, 409-447.

[Mc79] R. C. McOwen, The behavior of the Laplacian on weighted Sobolev spaces, Comm. Pure Appl. Math. 32 (1979), 783-795.

[Mi63] C. Misner, The method of images in geometrostatics, Ann. Physics 24 (1963), 102-117.

[MI96] V. Moncrief and J. Isenberg, A set of nonconstant mean curvature solutions of the Einstein constraint equations on closed manifolds, Classical Quantum Gravity 13 (1996), 1819-1847.

[Sc02] M. Schechter, Principles of functional analysis, Americal Mathematical Society, Providence, Rhode Island, 2002.

[ST] H. Smith and D. Tataru, Sharp local well posedness results for the nonlinear wave equation, To appear Ann. Math.

[Th87] J. Thornburg, Coordinates and boundary conditions for the general relativistic initial data problem, Classical Quantum Gravity 4 (1987), 1119-1131.

[Tr73] N. Trudinger, Linear elliptic operators with measurable coefficients, Ann. Scuola Norm. Sup. Pisa Cl. Sci. 27 (1973), 265-308.

[Wa84] R. M. Wald, General relativity, The University of Chicago Press, Chicago, 1984.

[YB80] J. W. York, Jr and J. Bowen, Time-asymmetric initial data for black holes and black hole collisions, Phys. Rev. D 24 (1980), no. 8, 2047-2056. 